\title{
Rapport Juin-Juillet 1999
}

\author{
J. C. Chanteloupe
}

August 18, 1999

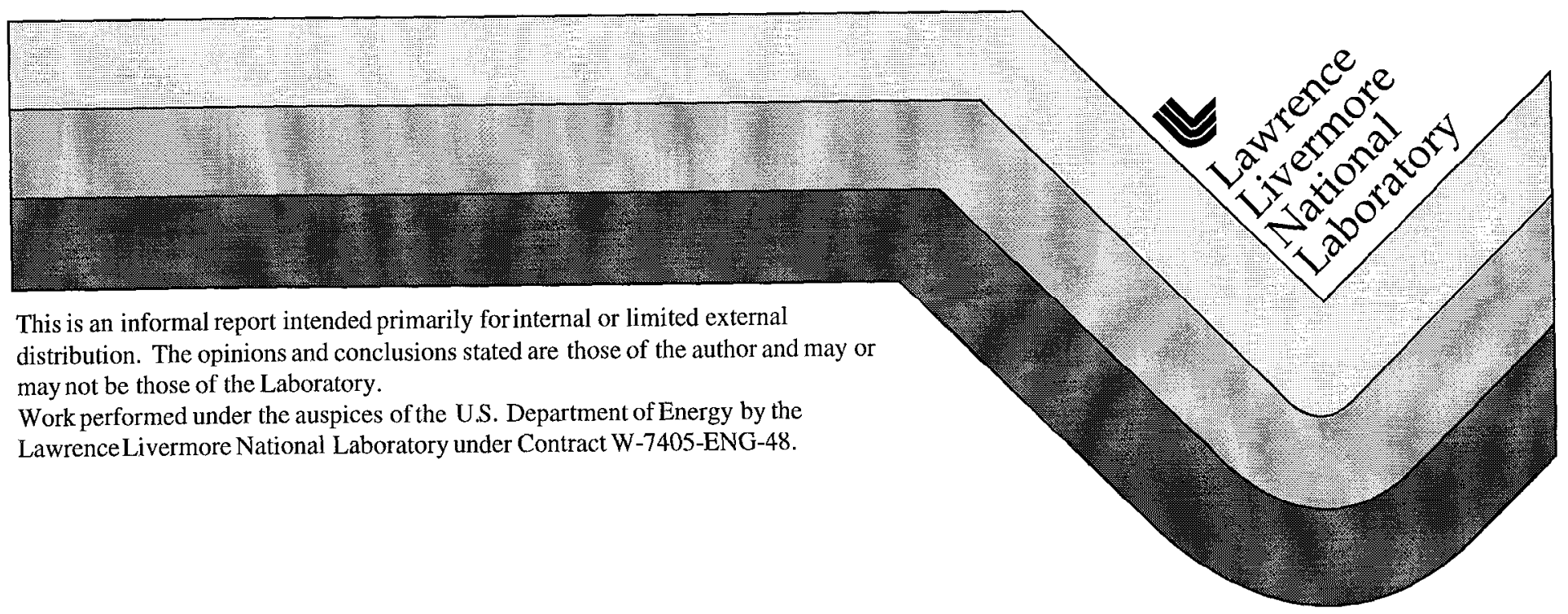




\section{DISCLAIMER}

This document was prepared as an account of work sponsored by an agency of the United States Government. Neither the United States Government nor the University of California nor any of their employees, makes any warranty, express or implied, or assumes any legal liability or responsibility for the accuracy, completeness, or usefulness of any information, apparatus, product, or process disclosed, or represents that its use would not infringe privately owned rights. Reference herein to any specific commercial product, process, or service by trade name, trademark, manufacturer, or otherwise, does not necessarily constitute or imply its endorsement, recommendation, or favoring by the United States Government or the University of California. The views and opinions of authors expressed herein do not necessarily state or reflect those of the United States Government or the University of California, and shall not be used for advertising or product endorsement purposes.

This report has been reproduced directly from the best available copy.

Available to DOE and DOE contractors from the Office of Scientific and Technical Information

P.O. Box 62, Oak Ridge, TN 37831

Prices available from (423) 576-8401

Available to the public from the

National Technical Information Service

U.S. Department of Commerce 5285 Port Royal Rd.

Springfield, VA 22161 


\section{June-July 1999 report}

This report describes the optical system which allows the delivery, in an efficient and homogeneous way, of the pump light from the diode arrays of the Mercury laser system described in the two previous reports. I will, first, describe the present pumping line; the description of the Advanced Pumping Design (APD) being given in the second part of this report. 


\section{Part one : Present pumping line}

I briefly described this system during my oral presentation last May in Limeil. The pumping line we are talking about is a catadioptric optical device: it is indeed based on the use of reflective optics (hollow ducts) as well as refractive (lenses, a dichroic plate and windows).

Figure 1 gives a longitudinal view in a vertical plan.

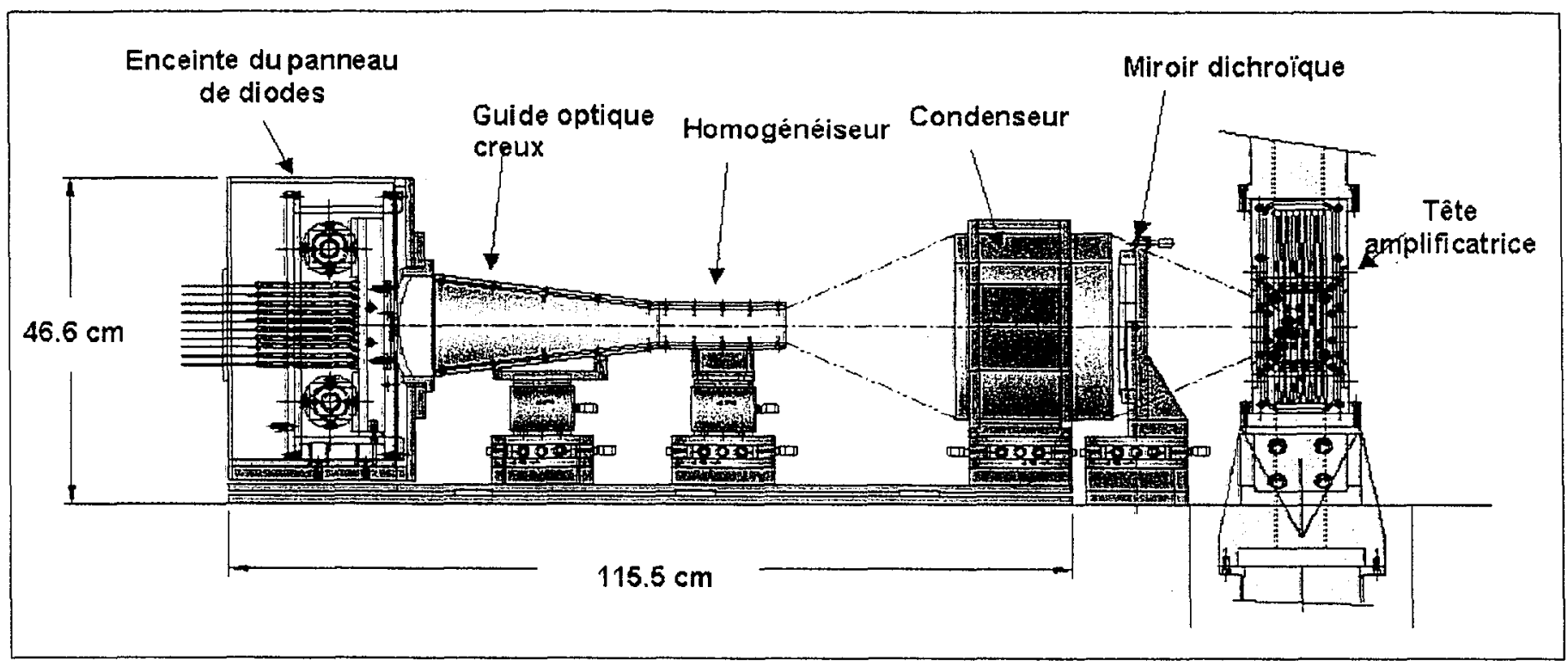

Figure 1: Mercury present pumping line. The yellow triangle shows the optical aperture of the condenser; the $900 \mathrm{~nm}$ pumping beam aperture being narrower.

Description of the constitutive elements of one of the two counter-propagative pumping lines of a Mercury amplifier head.

\section{$\underline{\text { II-1 Diode array }}$}

The diode array and its double enclosure appear on the left. Each array is made of 40 tiles, itself resulting from the association of 40 bars composed of 70 emitters (the diodes strictly speaking). Total output power should be $160 \mathrm{~kW}$ per array. The tiles are fixed on a copper backplane cooled by circulating hydrofluoroether (HFE7100) allowing to reach a minimal temperature of $-40^{\circ} \mathrm{C}$. In order to avoid any condensation, the primary enclosure is purged of any moisture by a nitrogen flux. 


\section{II-2 Hollow ducts}

The $40(10 \times 4)$ tiles are arranged in a $12 \times 17 \mathrm{~cm}^{2}$ rectangular area. The goal is to concentrate the light emitted by this spatially-incoherent extended source into a $3 \times 5 \mathrm{~cm}^{2}$ section (i.e. the aperture of a $\mathrm{Yb}: \mathrm{S}$-FAP slab). The difficulty arises from the fact one must not only perform the energy transport with the highest efficiency (transmission through the pumping line) but one also should obtain an angular distribution as narrow as possible along the optical axis. And these two constraints must be achieved when the pumping light reaches the first of the 7 slabs constituting the amplifier head. It is indeed a matter of being able to obtain a sufficient collimation to pump all the disks in an uniform way (or at least the 4 firsts since all 7 slabs are pumped from both ends and most of the pump energy is absorbed by the firsts ones).

The present design is based on the used of a pair of hollow ducts. The internal surfaces of these optical guides reflect the $900 \mathrm{~nm}$ light coming from the diodes with a $98.3 \%$ coefficient. Both ducts are made of four Aluminum plates which are polished, silver and dielectric coated. The first duct concentrates the light : it exhibits a truncated pyramidal shape (both ends have a rectangular section) and it will be designated, in this report, as the hollow lens duct or simply lens duct. The second duct has been designed to homogenize the energy transverse distribution coming out of the first duct : it is then called an homogenizer.

Whereas the lens duct acts also on the k-vector angular distribution of the $900 \mathrm{~nm}$ radiation, the homogenizer acts only on the rays spatial distribution. Its facing internal surfaces are indeed parallel and, a ray is, a priori, successively reflected only by such parallel surfaces in order to avoid any partial depolarization.

\section{II-3 Condenser}

Ideally, the gain media would have to be located just at the homogenizer output. But, Mercury is based on a colinear pumping and extraction scheme. And, in order to satisfy this constraint, the present design required a dichroic plate to be inserted somewhere along the optical path followed by the pumping radiation. Although it could have been conceivable to open a window in one of the panels of the ducts, it has been preferred to rely on the use of a large aperture dioptric condenser made of four lenses. Note that the window option would also have required the window to be dichroic (inducing actually an extra difficulty: to be able to achieve a high reflectivity at $900 \mathrm{~nm}$ for low incidence angles). 


\section{II-4 Dichroic plate}

This plate was initially supposed to work at an angle of incidence of $45^{\circ}$. Sample tests have not been able to demonstrate a high reflectivity at $1047 \mathrm{~nm}$ above $40^{\circ}$, consequently, the coating specifications were :

- Reflectivity@900nm : 0.25\% on both side for an angle of incidence varying from $0^{\circ}$ to $20^{\circ}$.

- Reflectivity @1047nm : 99.8\% for the extraction beam reflective side with an angle of incidence of $20+/-5^{\circ}$.

- Damage threshold : $30 \mathrm{~J} / \mathrm{cm}^{2}$ for a 3 ns laser pulse.

The plate was ordered with a size almost equal to the laser beam $(3 \times 5 \mathrm{~cm})$ projected footprint. This plate must be inserted across the pumping beam : dielectric layers consequently need to cover all the surface to reach the edges.

Unfortunately, recent experimental results show that the delivered plates generate several waves (i.e. micrometers) of phase-front distortions when used in a reflective mode. These are probably due to dielectric layer stress-induced surface deformations.

\section{$\underline{\text { II-5 Computer code }}$}

This pumping line has been initially developed by using commercially available ray-trace software (Opticad) used on PC. Opticad radiometry features allow us to predict intensity distribution patterns. The quality of these predictions has been checked by performing experimental measurements in the laboratory. Such a comparison requires about ten hours of computing time and 2 millions rays to be «launched » through the computer model of the pumping line. Rays are launched with a random distribution as well in the spatial domain (coordinates of the ray origin on the backplane) as in the angular domain (according to an energy distribution experimentally obtained for both fast and slow axis of the existing diodes) Obviously, such a computer model does not take into account interference/diffraction phenomena. Nevertheless, the about 100.000 emitters constituting each of the four arrays can be considered as being temporally incoherent with respect to each others. Moreover, the smallest spatially coherent area is much below a square millimeter. It is then a reasonable assumption to consider this source as being incoherent, or at least to neglect any possible interference pattern. 


\section{Part two : Advanced Pumping Design (APD)}

\section{II-1 Difficulties inherent to the present design}

Essentially two reasons lead us to reconsider the architecture described in Part one :

1/ - The withdrawal of a diode technology based on the use of $\mathrm{BeO}$ heatsink.

The back-up plan consists in using Silicon instead of BeO. Difficulties to meet dimensional tolerance specifications in machining $\mathrm{Si}$ heatsink are responsible for the new geometrical diode bar configuration. Present tiles are made up of 40 diode bars emitting in a direction normal $\left(90^{\circ}\right)$ to the tile plane. New tiles will be composed of 23 bars emitting at approximately $55^{\circ}$ from the normal axis. Brightness (W/ $/ \mathrm{cm}^{2} / \mathrm{sr}$ ) stays identical but two times more tiles are now required in order to achieve an equivalent output power, and moreover, a new backplane has to be designed.

$2 /$ - The use of refractive optics (dioptrics) decreases the overall transmission of the pumping line.

The present system exhibits 18 glass-air interfaces encountered by the pumping light: microlenses, primary enclosure window, secondary enclosure lens (=window), four lenses constituting the condenser, dichroic plate and, finally, amplifier head window. Moreover, as we have seen earlier, the dichroic plate is responsible for wave front distortions.

\section{$\underline{\text { II-2 Constraints to comply with when designing the APD }}$}

It appeared then to be required to propose a new architecture for the longitudinal pumping of Mercury. Constraints I was invited to take into account are as follows :

1/ To maximize the transmission between diodes and four firsts slabs.

2/ To obtain an intensity distribution as homogeneous as possible over the slab section $\left(3 \times 5 \mathrm{~cm}^{2}\right)$. 
3/ To decrease the number of interfaces and reject use of any dichroic optics.

4/ To take into account the new diode emission geometry in the APD.

5/ To manage the extraction beam multiplexing required in order to extract 100 Joules.

6/ To take into account optical damage thresholds during last amplifying pass and in the external multiplexing optical system.

7/ To localize ghosts in the APD .

8/ To keep in mind that the APD must be as scalable as possible in the framework of IFE.

I will come back, in the following sections, to the implications of some of these constraints.

\section{$\underline{\text { II-2-1 Transmission Efficiency }}$}

In order to evaluate the quality of light transmission ( $1^{\text {st }}$ constraint) for a specific APD geometry developed with Opticad, I evaluate the number of rays intercepting all seven slabs. Figure 2 shows a green curve obtained with the present catadioptric design as well as two curves corresponding to different APDs. I indeed proposed two kinds of tiles distribution on the backplane : an «I-shape » and a « C-shape » arrangement, hence the black and red curve captions.

It is very much desirable to make these curves as horizontal as possible (at least for the four firsts slabs). Satisfying this point, globally comes down to simultaneously concentrating rays emitted by the diodes and minimizing their $\mathrm{k}$-vectors angular distribution at the entrance of the amplifier head. Then, almost all rays encountering slab \#1 will also go through slab \#4. 


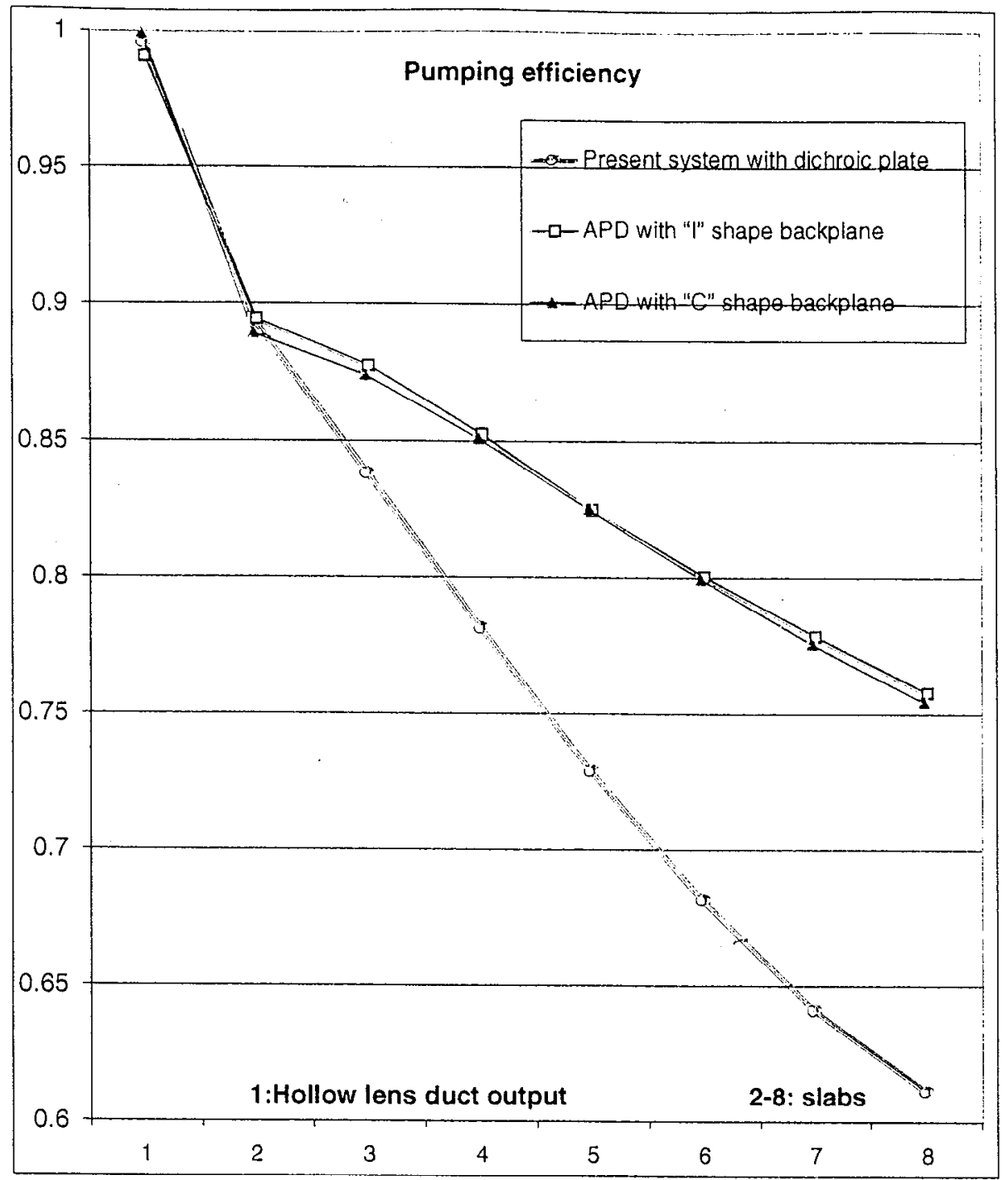

Figure 2: Number of rays encountering the homogenizer entrance and the 7 slabs constituting a Mercury amplifier head. This figure is divided by the total amount of rays initially emitted by the diode array. It gives rise to a value less than one. It appears that almost $10 \%$ of the diode light does not reach slab \#1 : the corresponding rays (the less efficiently collimated) are retro-reflected towards the diodes or refracted outside the head by the amplifier head window.

\section{II-2-2 Pumping homogeneity}

It is relatively uneasy to define a simple figure of merit estimating the capability of a specific APD computer model to satisfy constraint \#2.

Opticad radiometry features allow the recording of energy transverse distribution anywhere in the developed optical model. To do so, it is only required to place photographic films at the chosen location of the modeled APD. One can then get a spatial distribution of the fluence similar to what would be obtained with a real film; the only difference being the fact that this film does not absorb any energy. In some way, each ray only leaves a print of its path at a specific location on the film. It is then possible to add an unlimited (except by computing 
time) number of films in the modeled APD. Figures 3 and 3 bis give energy maps obtained at 8 different locations (homogenizer entrance and surface of each of the 7 slabs).

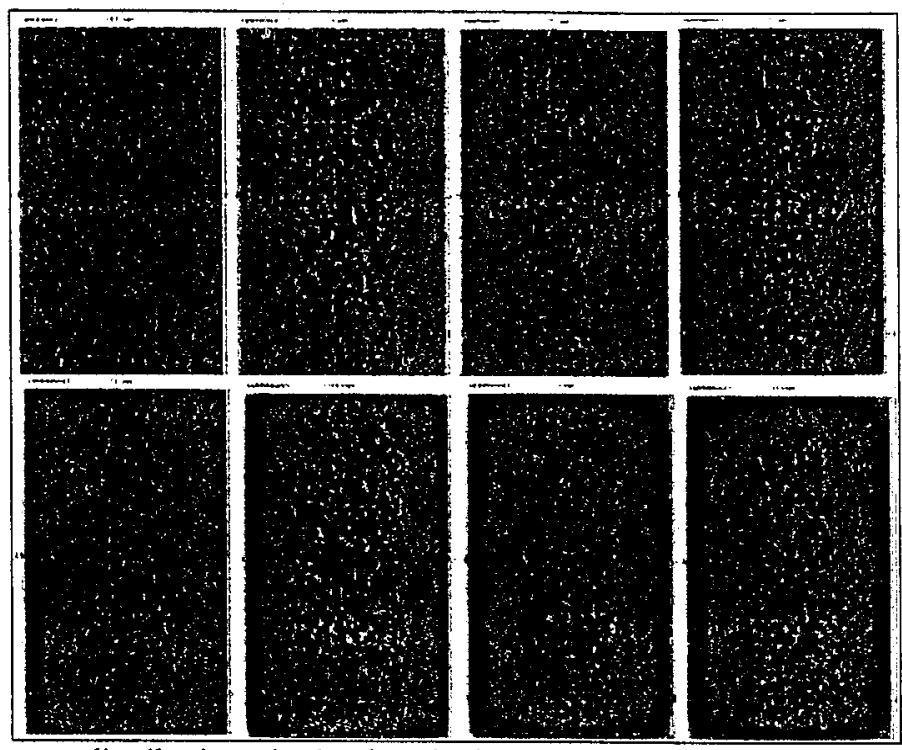

Figure 3 : Transverse energy distribution obtained at the homogenizer entrance and each of the 7 slabs location (for the APD). Film aperture is $3 \times 5 \mathrm{~cm}^{2}$. Pixel size is $0.5 \times 0.5 \mathrm{~mm}^{2}$, corresponding to $60 \times 100$ pixels. Two millions rays, each carrying an amount of energy equal to unity, are used ; this gives an average of 333 rays intercepting a pixel. Color scale is arbitrary. Diode tiles are distributed here over a "I" shaped backplane.

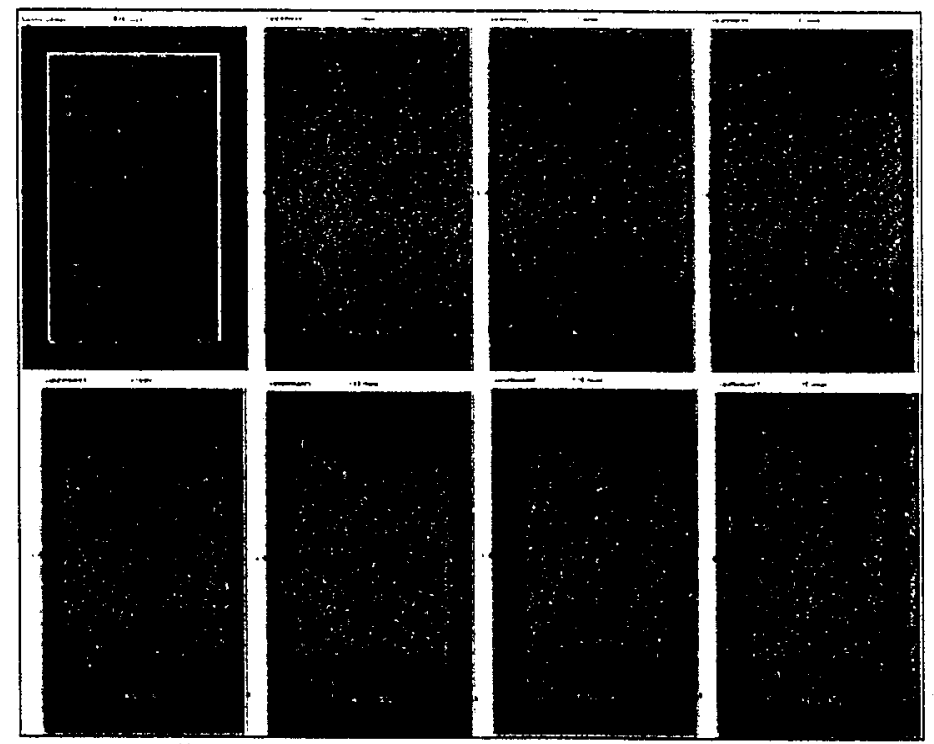

Figure 3bis: Transverse energy distribution obtained at the homogenizer entrance and each of the 7 slabs location (for the present configuration based on the use of the dichroic plate and the condenser). Film aperture is $3 \times 5 \mathrm{~cm}^{2}$ (except for the first one : $4 \times 6 \mathrm{~cm}^{2}$ ). Color scale is arbitrary (red=energy minimum, blue=energy maximum). Transverse energy distribution is more homogeneous here than on figure 3 . The reason for that being the absence of any hole in the diode backplane. 
Integrating over the film area, one obtains a value which, divided by 2 million (total amount of energy coming out from the diodes), gives efficiencies $(<1)$ used to plot the curves of figure 2. Figure 4 gives a partial 3D view of a homogenizer and the seven slabs.

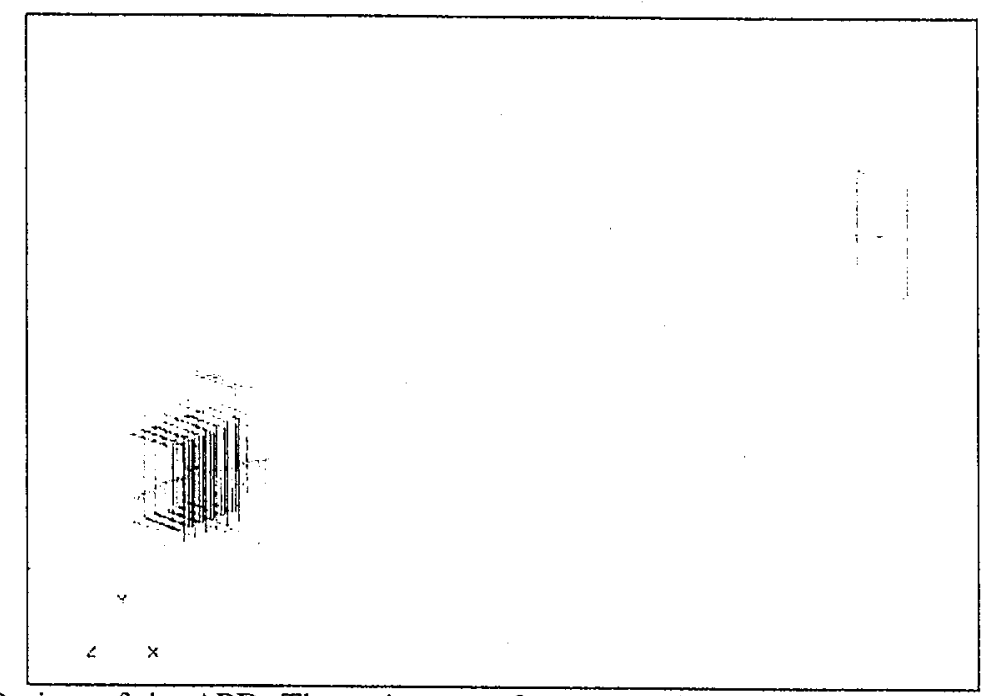

Figure 4 : Partial 3D view of the APD. The trajectory of a ray coming out from a diode is represented in dark blue. It goes first through the film located at the entrance of the homogenizer (vertical rectangle on top-right) ; is reflected laterally three times inside the homogenizer, goes through the amplifier head window (blue green), then, finally, it encounters the $7 \mathrm{Yb}: \mathrm{S}$-FAP slabs on the surface of which is located a film. Graduations represented in the $(\mathrm{xz})$ plane are separated by $1.5 \mathrm{~cm}$.

In order to roughly compare the ability of two models to achieve a homogeneous energy distribution, I qualitatively study energy maps of figures 2 and more specifically those corresponding to slabs 1 and 4 for which I plot vertical and horizontal profiles.

From a general point of view, the APD allows a better concentration of the k-vector distribution (curves are more horizontal on figure 2) but the homogeneity of the transverse energy distribution is notably affected by the presence of the aperture allowing a path for the extraction laser beam between the two half-backplanes.

\section{II-2-3 Diodes emission geometry}

The new geometry of the diode emission (constraint \#4) requires us to reconsider the tiles arrangement. First, the tile support must rotate in order to compensate for the non perpendicular emission of the diodes (figure 5). 


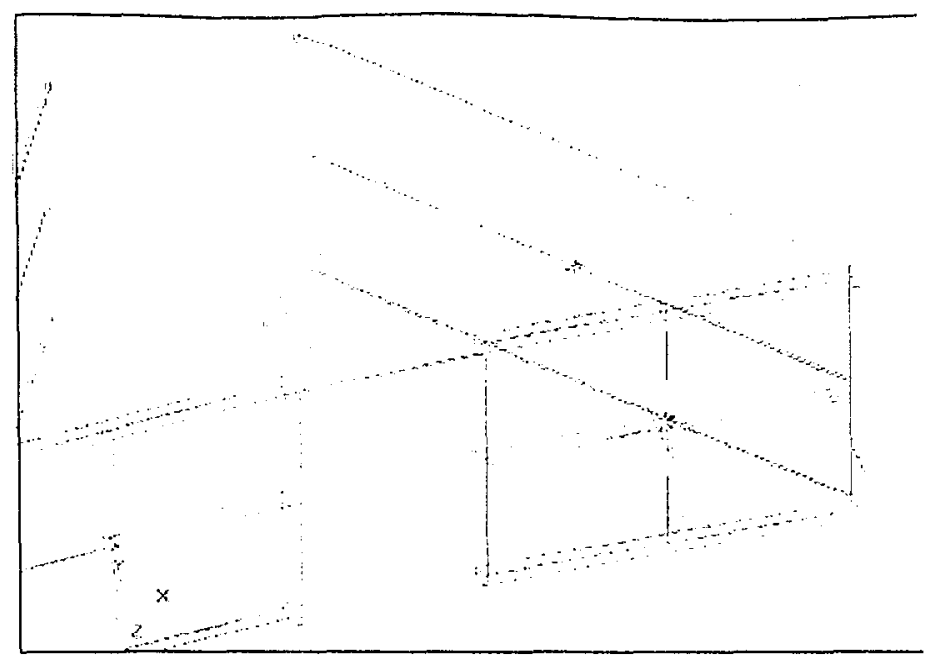

Figure 5: 3D representation of two half backplanes made of $5 \times 7=35$ tiles each (in the case of the " $\mathrm{I}$ " shape tile arrangement shown on figure 6). Both navy blue trajectories are associated with a pair of rays emitted by two diodes. They originate in a plane rotated by $55^{\circ}$ with respect to the optical axis of the square aperture focalization lens (green blue) shown at the foreground.

Moreover, in order to obtain a similar power with the new 23-bars tiles, a number at least equal to $70(\sim 10 \times 4 \times 40 / 23)$ is required. My calculations converged toward two tile distributions :

$1^{\text {st }}$ case : Two rectangular half backplanes (hence the « $\mathrm{I} »$ shape) of 35 tiles each ( 5 columns of 7 tiles. They are separated by 6 to 7 centimeters in order to let a path for the angularly multiplexed extraction beams (figure 6).

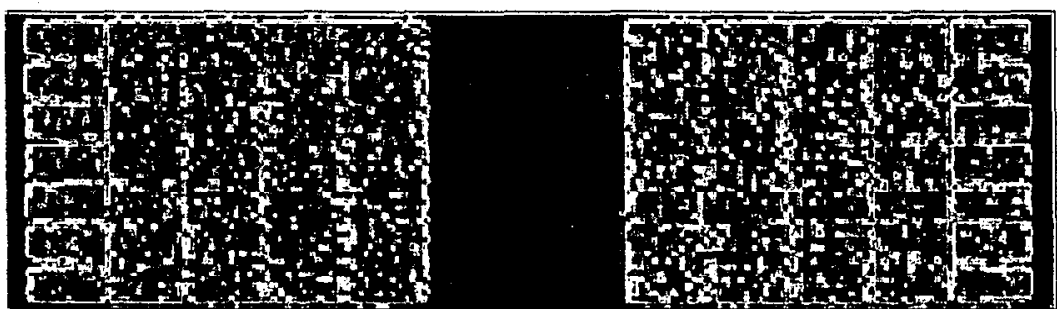

Figure 6: This energy map is obtained using a tilm located in a plane normal to the extraction beam optical axis. Red means absence of energy. One can distinguish 10 columns of 7 tiles. The two columns located on the far right and left are very close to the film. The tiles they are made of consequently appear clearly separated from each other. This is not the case for the internal columns because these are located 5 times further away from the film and the fact that the diode divergence is not null explains why gaps between tiles are filled with rays coming from adjacent diodes. Central aperture $(6.6 \mathrm{~cm}$ wide) allows room for the extracted beam path (a $3 \mathrm{~cm}$ width plus extra space for a 4 passes multiplexing) and takes into account 1 to $2 \mathrm{~cm}$ of mechanical mounts. Total aperture of this film is $-9 \times 32 \mathrm{~cm}^{2}$. 
$2^{\text {nd }}$ case : Two half-backplanes of 34 tiles each arranged in a $« \mathrm{C} »$ shape and facing each other (figure 7). Such an arrangement of tiles offers a more compact source geometry and leads to a better homogeneity in the energy deposition at the slabs level. The drawback of this diode distribution concerns the ability to uniformly cool all 34 tiles (in order to have the same emission wavelength for all tiles). This « $\mathrm{C} »$ shape makes this goal, a priori, less obvious to achieve that with the «I » shape arrangement.

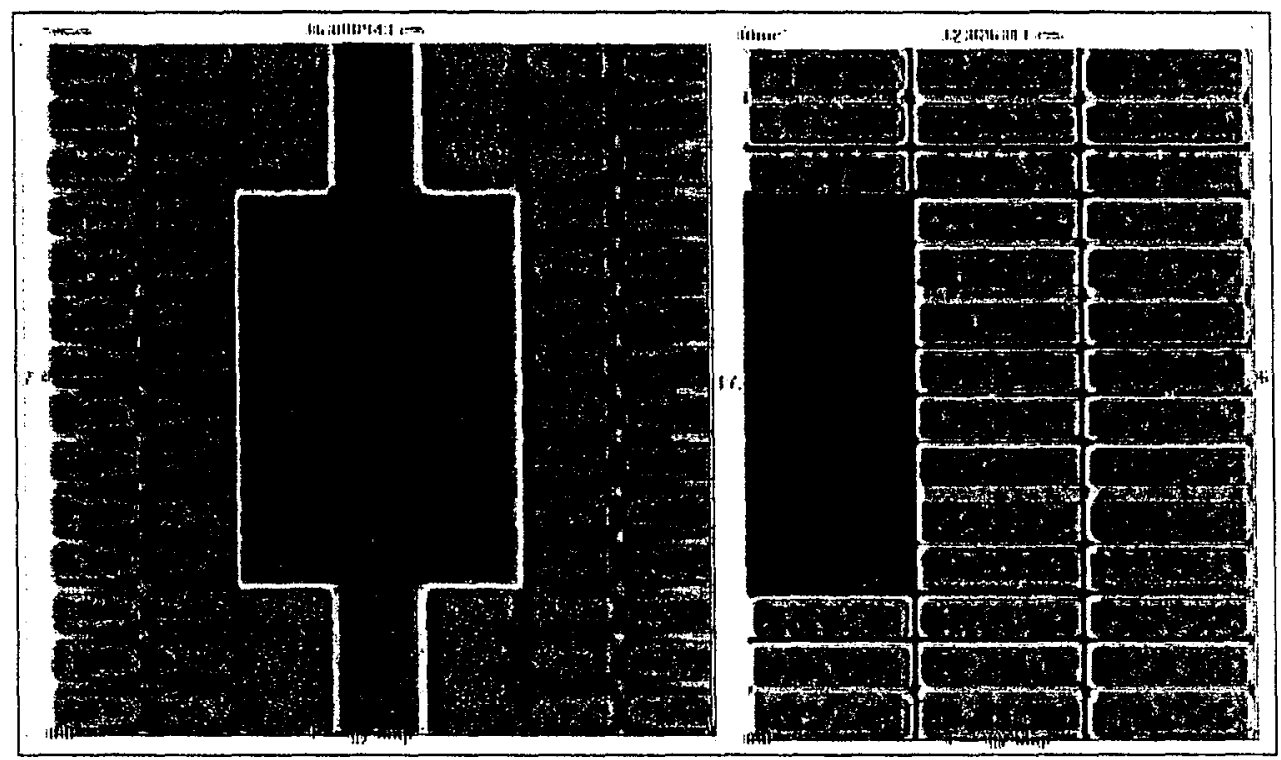

Figure 7 : The energy map on the right shows the arrangement of the 34 tiles $\left(1 \times 4 \mathrm{~cm}^{2}\right.$ each) when observed in the plane of the $C$ » shaped half backplane. The left map was obtained by use of a film located in a plane normal to the extraction beam optical axis. Central aperture $\left(6.6 \times 10 \mathrm{~cm}^{2}\right)$ allows room for the extracted beam path (a $3 \mathrm{~cm}$ width plus extra space for a 4 path multiplexing) and takes into account 1 to $2 \mathrm{~cm}$ of mechanical mounts. Total aperture of this film is $\sim 16 \times 17 \mathrm{~cm}^{2}$.

\section{II-2-4 Angular multiplexing}

I already mentioned that the 4 passes scheme, required in order to extract 100 joules, was based on an angular multiplexing of the extraction beam (note that an alternate - but costly solution could be based on the use of gas cooled plasma electrode Pockel cells). This angular distribution of trajectories is responsible for moving diodes away from the extraction optical axis. It also implies a widening of the entrance section of the homogenizer. Consequently, this duct now does not only homogenize the pumping light distribution : it also acts on its $\mathrm{k}$-vector distribution.

The multiplexing system is based on the use of an imaging telescope coupled to a tilted mirror. The horizontal angular separation is defined by injecting the beam coming from the front-end with a non-zero angle with respect to the amplifier heads optical axis. Several 
geometries are conceivable; the option currently studied is based on the use of mirrors (or prisms) located in the focal area of the imaging telescope (I believe a similar solution has been chosen for the LMJ). 
The $6^{\text {th }}$ constraint is by far the most critical of all. The Mercury laser chain is based on image relay between amplifier heads. Optics located at positions too far from relay planes would be exposed to unacceptable level of fluences. This is especially true after the last amplification stage (100 J, $10 \mathrm{~ns}, 10 \mathrm{~Hz}, 15 \mathrm{~cm}^{2}$ ), but remains valid at the level of the multiplexing mirror M1 (figure 8) after the second pass too. Taking into account intensity modulations due to the present Yb:S-FAP slab optical quality as well as the free space propagation, it does not appear conceivable to place any optics further than a few centimeters from the amplifier head (image plane). The first lens LO belonging to all three telescopes (injection, feed-back and output) has then to be located as close as possible to this plane. Also, the second lens of the output telescope (L2) must have a focal length longer than LO's in order to reduce the peak intensity of the modulations seen by L2 (this lens being indeed located far from any image plane). Let me finally remind the reader that, at this moment, no spatial filtering - even at very high spatial frequency - seems to be conceivable due to fluence constraints $(100 \mathrm{~J} / 10 \mathrm{~Hz})$.

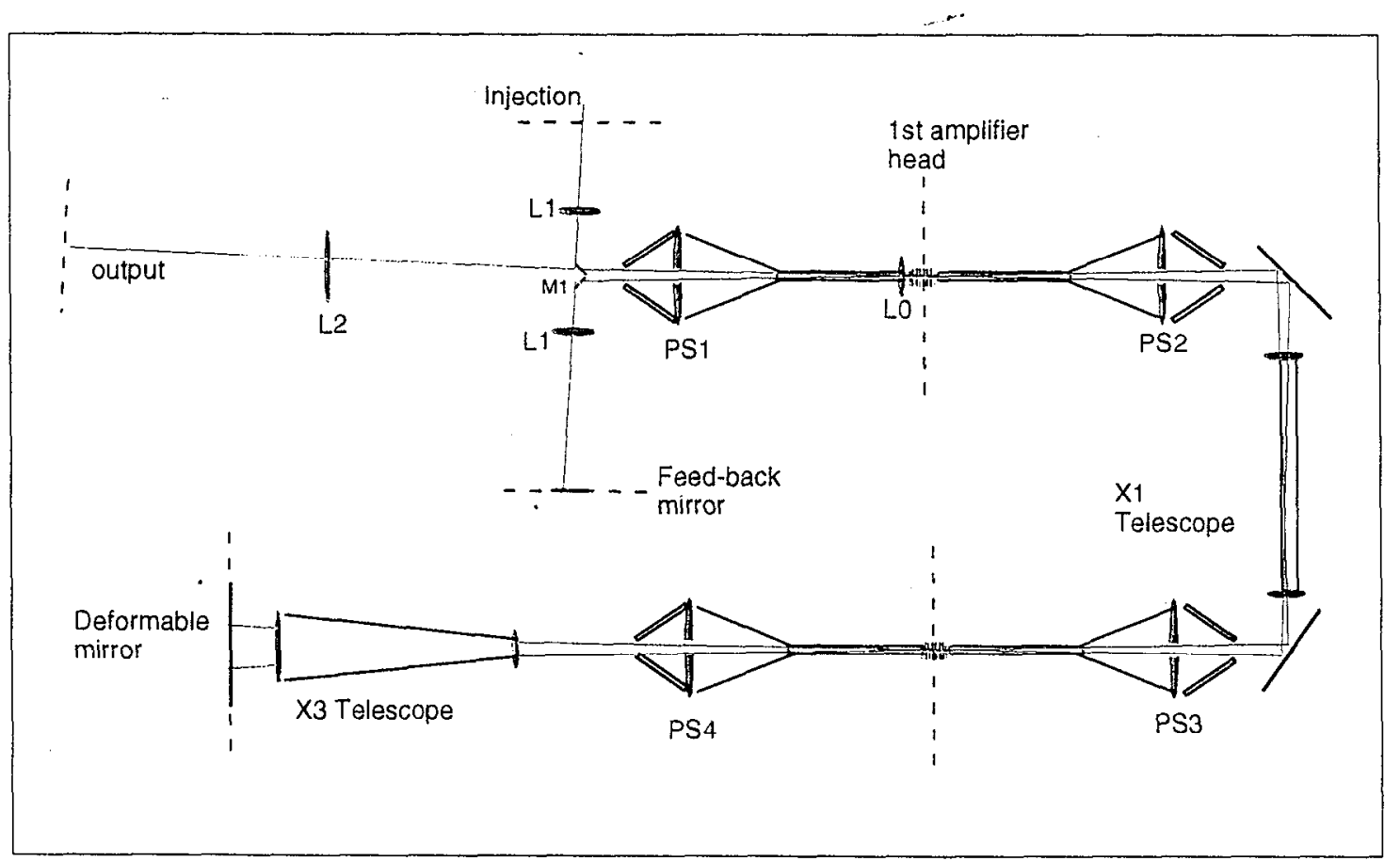

Figure 8: APD schematic overview in an horizontal plane. Dashed segments are associated to the six image planes of this system. Red lines represent optical axis followed by the $1047 \mathrm{~nm}$ extraction beam for its four angularly multiplexed passes (only two trajectories appear because the two other are located in an identical vertical plane). The two amplifier heads are represented by the seven green slabs. Finally, red rectangles symbolize diode half backplane rotated by 55 degrees. 
Figure 8 shows four pumping systems (PS1 to 4), each made of a lens (with a hole at its center in the case of the « $\mathrm{C} »$ shape half-backplanes), a hollow lens duct and an homogenizer. PS2,3 and 4 are identical. For PS1, the amplifier head flat window is replace by the LO lens.

\section{II-3 Proposed Models for the APD}

During these past weeks, I have studied several different geometrical configurations. The best trade-off of efficiency/homogeneity/compactness is associated with the design shown on the figure below. An important point still to be checked concerns the ghost analysis required by the presence of a lens in place of the amplifier head window ( $7^{\text {th }}$ constraint).

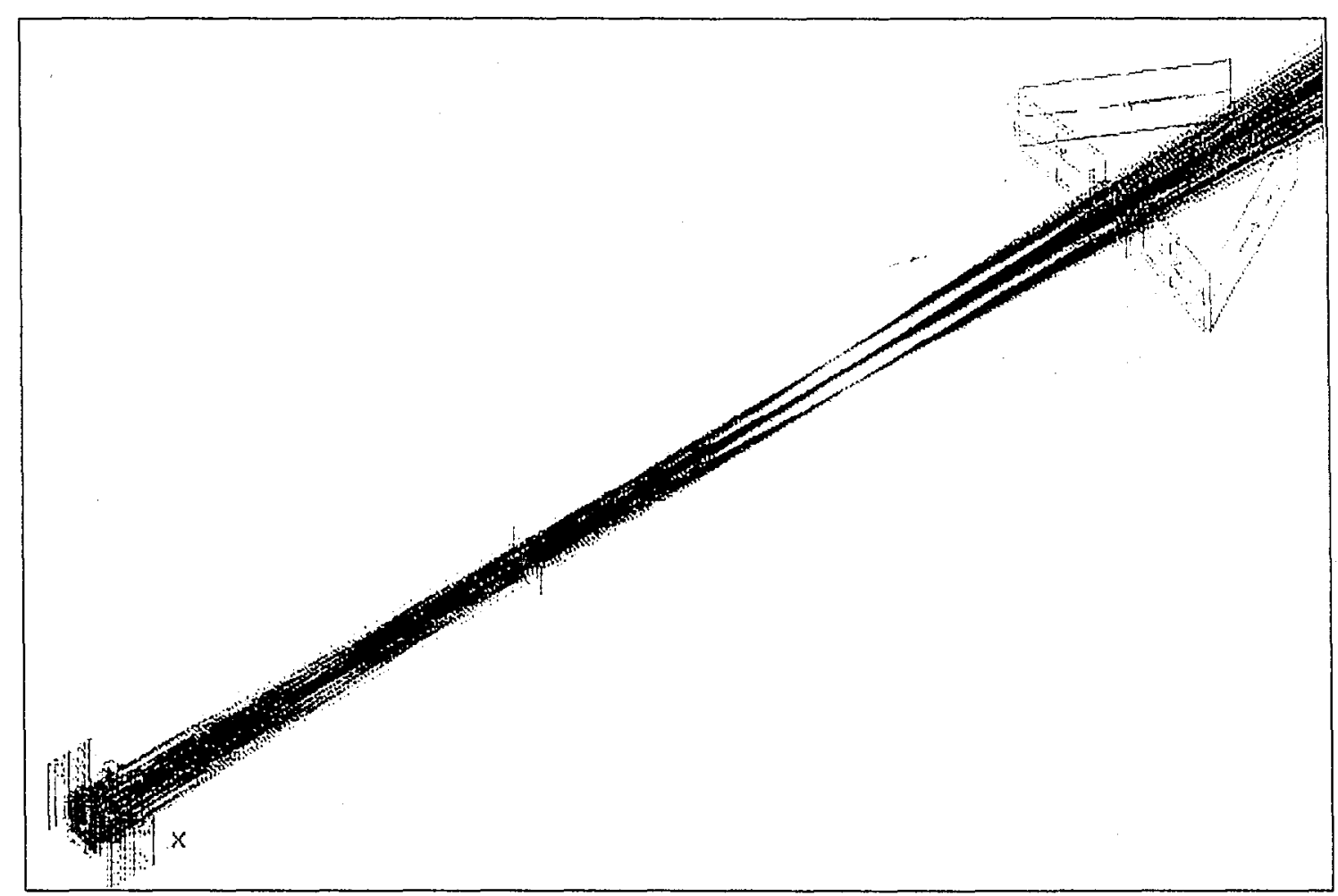

Figure 9: 3D view of one of the pumping systems (PS1) constituting the Mercury APD. Blue rays represent this time the extraction beam radiation at $1047 \mathrm{~nm}$. The four angularly multiplexed passes can be seen on this scheme, especially at the focal plane level where the rays are geometrically separated. Note that these focal spots are localized inside the hollow lens duct. This duct (together with the homogenizer) will then have to be inserted into a vacuum vessel. A priori, this task is going to increase the complexity of the overall system (APD) since it is indeed out of question to place the diode backplanes in vacuum. 